\title{
Geometrically Exact Theory of Contact Interactions-Applications with Various Methods FEM and FCM
}

\author{
Alexander Konyukhov ${ }^{1,2}$ \\ ${ }^{1}$ Institute of Mechanics, Karlsruhe Institute of Technology, Karlsruhe, Germany \\ ${ }^{2}$ Department of Strength of Constructions, Kazan National Technical University, Kazan, Russia \\ Email: Alexander.Konyukhov@kit.edu
}

Received 15 July 2015; accepted 19 August 2015; published 26 August 2015

\begin{abstract}
Geometrically exact theory of contact interactions is aiming on the development of the unified geometrical formulation of computational contact algorithms for various geometrical situations of contacting bodies leading to contact pairs: surface-to-surface, curve-to-surface, point-to-surface, curve-to-curve, point-to-curve, point-to-point. The construction of the corresponding computational contact algorithms is considered in accordance with the geometry of contacting bodies in covariant and closed forms. These forms can be easily discretized within various methods such as the finite element method (FEM), the finite discrete method (FDM) independently of the order of approximation and, therefore, the result is straightforwardly applied within any further method: high order finite element methods, iso-geometric finite element methods etc. As particular new development it is shown also the possibility to easy combine with the Finite Cell Method.
\end{abstract}

\section{Keywords}

Closest Point Projection Procedure, Curvilinear Coordinate System, Covariant Derivative and Linearization, Finite Cell Method

\section{Introduction}

Computational contact mechanics has become a separated branch of computational mechanics during the last decades. Modeling of contact interactions became standard in numerous finite element software packages available for engineers. Various aspects of the numerical solution such as various enforcements of contact conditions, or the possibility to apply high order and iso-geometric type of approximation have been considered. One of the important aspects, even though being obvious for everyone,- - the consistent geometrical treatment of contactis often remaining hidden inside the computational algorithm. Contact interaction from a geometrical point of view can be seen as the interaction between deformable surfaces possessing various geometrical features such as surfaces, edges and vertexes, therefore, geometrical approaches can be exploited. Three major geometrical situations can be identified: surface-to-surface, curve-to-curve and curve-to-surface contact. Numerous publications 
are devoted to development of various numerical aspects of the first two contact pairs: we refer here to the major monographs only. Thus, Laursen [1] describes many numerical methods for surface-to-surface contact, Wriggers [2], describes numerical algorithms for both surface-to-surface and beam-to-beam (or geometrically curve-tocurve) situations and Litewka [3] specially studied in detail beam-to-beam contact. A unified geometrical approach for various geometrical situations forming a basis of geometrically exact theory of contact interaction is described in the monograph of Konyukhov and Schweizerhof [4] and on the introductory level with programming examples in the monograph of Konyukhov and Izi [5].

The geometrically exact theory is straightforwardly applicable together with the isogeometric finite elements applications in computational contact mechanics-a comprehensive review of the state of the art is presented in De Lorenzis, Wriggers and Hughes [6]. The current contribution is aimed on showing the unified possibility of various applications within the Finite Element Method as well as within the relatively novel Finite Cell Method.

\section{Basis of the Geometrically Exact Theory-Solution of the Closest Point Projection Procedure}

\subsection{Various Contact Pairs and Corresponding Coordinate System}

It is easy to construct a model contact problem with two bodies possessing smooth surfaces as well as various geometrical features such as edges and vertexes-an example of this is an impact of two swords shown in Figure 1. Considering all possible geometrical situations in which both swords can contact each other, the following hierarchical sequence of contact pairs is appearing:

1. Point-To-Point contact pair;

2. Point-To-Curve contact pair;

3. Point-To-Surface contact pair;

4. Curve-To-Curve contact pair;

5. Curve-To-Surface contact pair;

6. Surface-To-Surface contact pair.

In order to construct a numerical algorithm for a certain contact pair, first of all, it is identified that the distance between contacting bodies is a natural measure of the normal contact interaction. The procedure is introduced via the Closest Point Projection (CPP) procedure, requiring the minimization of the distance between a selected point $r_{S}$ on the one surface, called "slave" surface, and a surface $\rho\left(\xi^{1}, \xi^{2}\right)$ on which a specific coordinate system is set up:

$$
\left\|r_{S}-\rho\left(\xi^{1}, \xi^{2}\right)\right\| \rightarrow \min .
$$

Solution of the CPP procedure requires the differentiability of the function representing the parameterization of a surface of the contacting body. Namely, analysis of the existence and uniqueness for the CPP procedure allows then to classify all types of all possible contact pairs, see details for surfaces in [7] and for curves in [8].

\subsection{Starting Point-Surface-To-Surface Contact Pair}

A Surface-to-Surface contact pair is described via the well known "master-slave" contact algorithm based on the CPP procedure onto the surface in Equation (1). This projection allows to define a coordinate system, see in Figure 2, as follows:

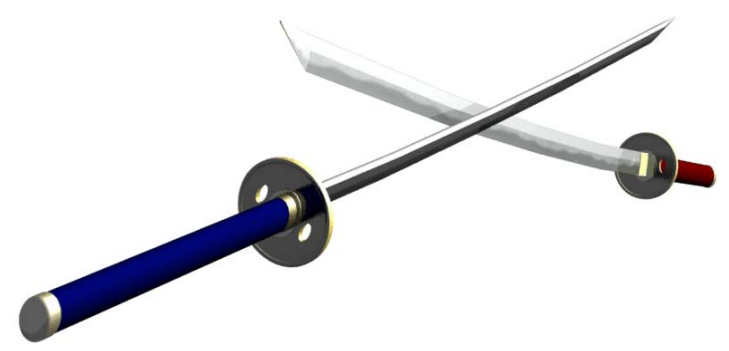

Figure 1. Impacting swords as an example in which all contact pairs are realized: Surface-To-Surface, Curve-To-Curve, Curve-To-Surface etc. 


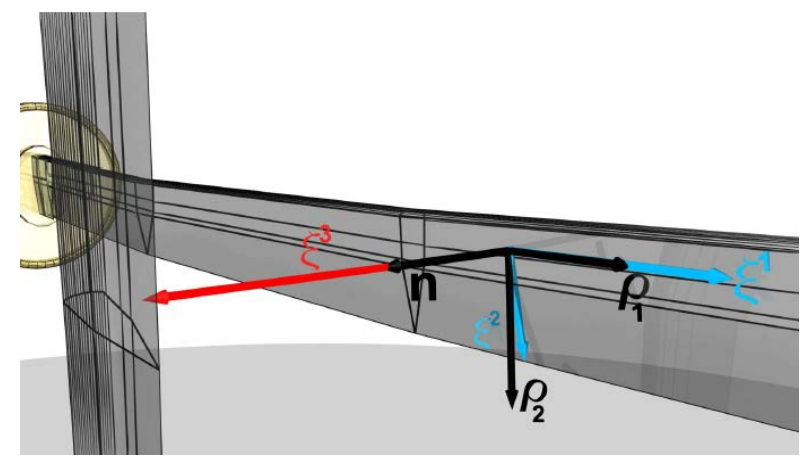

Figure 2. Surface-To-Surface contact pair and corresponding surface coordinate system.

$$
r_{S}\left(\xi^{1}, \xi^{2}, \xi^{3}\right)=\rho\left(\xi^{1}, \xi^{2}\right)+\xi^{3} n\left(\xi^{1}, \xi^{2}\right)
$$

The vector $r_{S}$ is a vector for the "slave" point, $\rho\left(\xi^{1}, \xi^{2}\right)$ is the parameterization of the "master" surface with Gaussian convective coordinates $\xi^{1}, \xi^{2}, n\left(\xi^{1}, \xi^{2}\right)$ is a unit normal to the surface. Equation (2) describes, in fact, a coordinate transformation in which convective coordinates are used to measure the contact interaction: $\xi^{3}$ is a penetration, $\Delta \xi^{1}, \Delta \xi^{2}$ are measures for tangent interaction. The algorithm is applied only in the allowable domain for the surface CPP procedure, i.e. in the domain in which the solution of the CPP procedure (1) exists and is unique. This allowable projection domain for 3D case can be constructed for utmost C1-continuous surfaces. If the surfaces contain edges and vertexes then the CPP procedure should be generalized in order to include the projection onto edges and onto vertexes. The main idea for application for the contact is then straightforward - the CPP procedure corresponding to a certain geometrical feature gives a rise to a special, in general, curvilinear 3D coordinate system. This coordinate system is attached to a geometrical feature and its convective coordinates are directly used for further definition of the contact measures. Thus, all contact pairs should be described in the corresponding local coordinate system. The requirement of the existence for the generalized CPP procedure leads to the transformation rule between types of contact pairs according to which the corresponding coordinate system is taken. Thus, the all contact pairs can be uniquely described in most situations.

\subsection{Curve-To-Curve Contact Pair}

The Curve-To-Curve (CTC) contact pair requires the projection on both curves, therefore, there is no classical "master" and "slave" and both curves are equivalent. For the description one of the two coordinate systems (Serret-Frenet coordinate system) can be assigned to the $i$-th curve, see in Figure 3:

$$
\begin{gathered}
\rho_{2}\left(s_{1}, r, \varphi_{1}\right)=\rho_{1}\left(s_{1}\right)+r e_{1}\left(s_{1}, \varphi_{1}\right) ; \quad e_{1}=v_{1} \cos \varphi_{1}+\beta_{1} \sin \varphi_{1} 1 \leftrightarrow 2, \\
e_{1}=v_{1} \cos \varphi_{1}+\beta_{1} \sin \varphi_{1} \quad 1 \leftrightarrow 2 .
\end{gathered}
$$

Here, the vector $\rho_{2}\left(s_{1}, r, \varphi_{1}\right)$ is a vector describing a contact point of the second curve, $\rho_{1}\left(s_{1}\right)+r e_{1}\left(s_{1}, \varphi_{1}\right)$ is a parameterization of the first curve; $e_{1}$ is a unit vector describing the shortest distance and is written via the unit normal $v_{1}$ and bi-normal $\beta_{1}$ of the first curve. Equation (3) describes the motion of the second contact point in the coordinate system attached to the first curve. The description is symmetric with respect to the choice of the curve 1 and 2. The convective coordinates used as measures: $r$-for normal interaction for both curves; $s_{i}$-for tangential interaction and $\varphi_{i}$-for rotational interaction of the $i$-th curve. The Curve-To-Curve contact pair can be used to model contact between edges of solid bodies as shown in Figure 3, see in [8] as well as to model contact between curvilinear beams and ropes, see applications in [9].

Remark 1. Representation for Curve-To-Curve contact in Equation (4) is possible until tangent $\tau_{1}$ and $\tau_{2}$ are not parallel. In the case of parallel tangents some difficulties are arising due to ill-condition of matrices and due to multiplicity of solution in the case of parallel straight lines. A special treatment is necessary to resolve this problem of parallel tangents. 


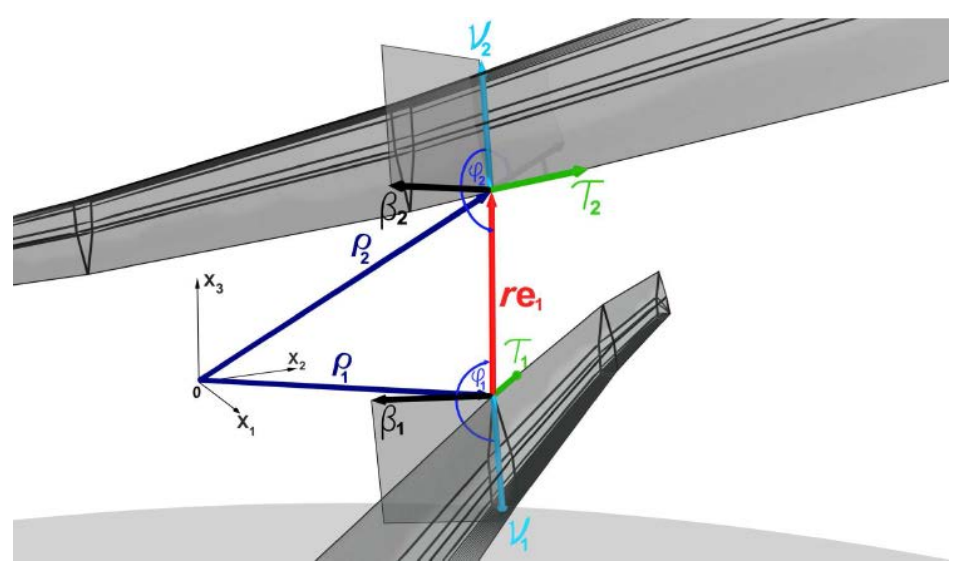

Figure 3. Curve-To-Curve contact pair and corresponding Serret-Frenet coordinate system.

\subsection{Curve-To-Surface Contact Pair}

A special development is necessary to describe a Curve-To-Surface (CTS) contact pair precisely from the kinematic point of view. Contact between a curve (geometrically representing either a rope, or a beam, or an edge of the solid) and a surface can be described by various approaches:

a) a special combination of Curve-To-Curve (CTC) and Segment-To-Analytical-Surface (STAS) Algorithm;

b) a special consideration in the Darboux basis.

\subsubsection{A Special Combination of Curve-To-Curve and Segment-To-Analytical-Surface Algorithm}

This type of contact, in case of rigid surfaces, is the combination of the Surface-To-Analytical-Surface and the Curve-To-Curve contact kinematics, see in [10]. All kinematic parameters are formulated dually in the surface coordinate system Equation (2) and in the following Serret-Frenet curve coordinate system Equation (3). Kinematic relationships during contact can be obtained dually considering the relative velocity of the contact point during contact interaction in both coordinate systems Equations (2) and (3). An auxiliary vector $g=\tau \times n$ is necessary to identify the dragging direction of the curve with regard to the surface, see Figure 4. Kinematic relations during contact can be obtained dually considering the relative velocity of the contact point during contact, thus normal, pulling and dragging relative velocities are derived:

$$
\begin{aligned}
v_{e}=\dot{\xi}^{3}=\dot{r}= & \left(v_{s}-v\right) \cdot n=\left(v-v_{s}\right) \cdot e-\text { normal relative velocity, } \\
& v_{\tau}=-\left(\rho_{i} \cdot \tau\right) \dot{\xi}^{i} \text {-pulling relative velocity, } \\
& v_{g}=-\left(\rho_{i} \cdot g\right) \dot{\xi}^{i} \text {-dragging relative velocity. }
\end{aligned}
$$

The contact forces are represented dually in both surface and curve coordinate systems as:

$$
\begin{gathered}
N_{\text {curve }}=N_{\text {surface }} \text {-normal contact force, } \\
T=-T^{i}\left(\rho_{i} \cdot \tau\right) \text {-pulling contact force, } \\
G=-T^{i}\left(\rho_{i} \cdot g\right) \text { - dragging contact force, }
\end{gathered}
$$

where $T^{i}$ are contravariant components of the tangent force in the surface coordinate system,

\subsubsection{A Special Consideration in the Darboux Basis}

In this approach, see details in [11], all kinematic parameters are described in the Darboux basis, introduced as the combination of both Gaussian surface basis vectors and Serret-Frenet basis. Both sets of basis vectors, from the surface $\left(\rho_{1}, \rho_{2}, n\right)$, and from the curve $(\tau, v, \beta)$ are used to define a new basis: a unit tangent of the curve $\tau$ is lying in the tangent plane to the surface and a unit normal to the surface $n$, see Figure 5. In addition a vector $g$ called the tangent normal is defined via the cross-product $g=\tau \times n$. Contact conditions for the rope 


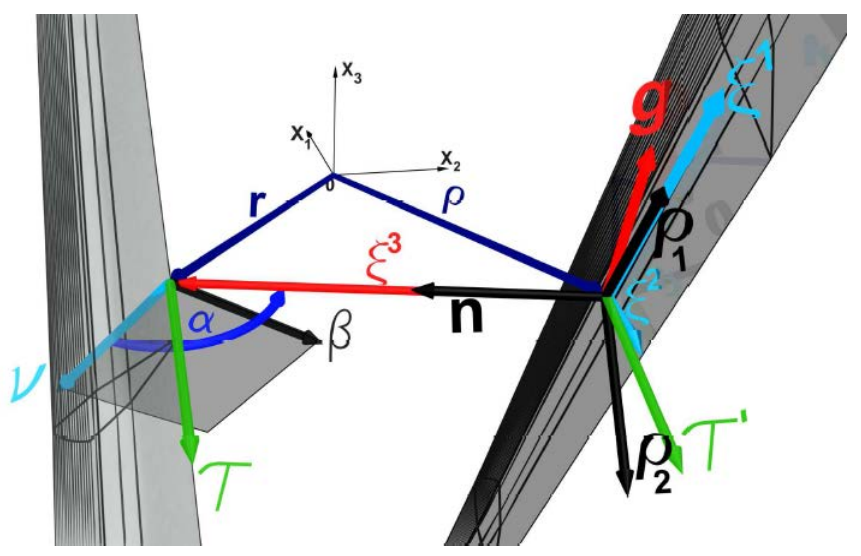

Figure 4. Curve-To-Surface contact pair. Special consideration in both surface and curve coordinate system.

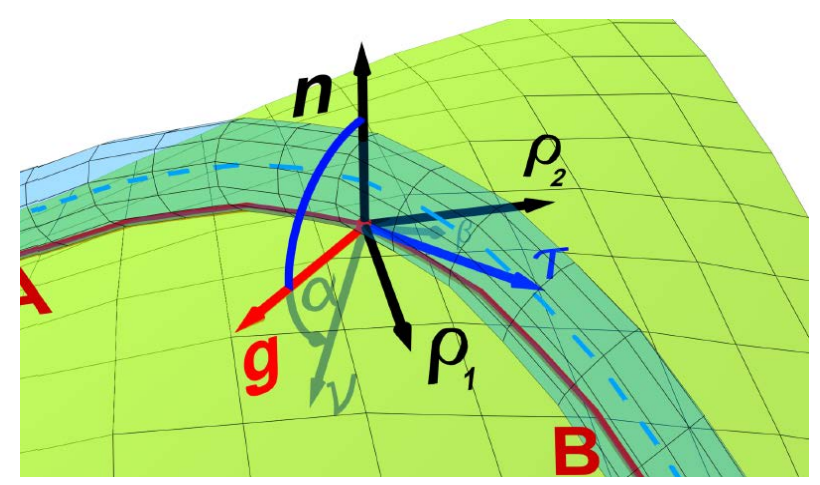

Figure 5. Curve-To-Surface contact pair. A special consideration in the Darboux coordinate system.

on a surface are formulated via constraint equations in the Darboux basis. These conditions can be formulated as Karush-Kuhn-Tucker (KKT) conditions, see details in [11].

\section{Weak Formulation for Contact Pairs in Corresponding Coordinate System and Their Linearization}

\subsection{Surface-To-Surface Contact Pair}

The weak formulation for the Surface-To-Surface contact pair is constructed in the surface coordinate system, Equation (2). First, the contact force/stress vector for the slave part is expressed in the local coordinate system on the master surface and is split into a normal and into a tangential part

$$
\mathbf{T}_{\text {slave }} \equiv \mathbf{T}=N n+T^{i} \rho_{i} .
$$

The weak form is formulated as

$$
\delta W_{c}=\int_{s} N \delta \xi^{3} d s+\int_{s} T_{j} \delta \xi^{j} d s=\int_{s} N\left(\delta r_{s}-\delta \rho\right) \cdot n d s+\int_{s} T_{j} a^{i j}\left(\delta r_{s}-\delta \rho\right) \cdot \rho_{i} d s .
$$

The linearized expression which is necessary for the tangent matrix is derived via the covariant derivation in the surface coordinate system, Equation (2), see details in [4] [12]. Exemplarily, the tangent matrix in the case normal non-frictional contact is shown

$$
\begin{gathered}
L\left(\delta W_{c}^{T}\right)=L\left(\int N \delta \xi^{3} d s\right)=\int_{s} \varepsilon_{N}\left(\delta r_{s}-\delta \rho\right) \cdot(n \otimes n)\left(v_{s}-v\right) d s \\
-\int_{s} N\left(\delta \rho_{, j} \cdot a^{i j}\left(n \otimes \rho_{i}\right)\left(v_{s}-v\right)+\left(\delta r_{s}-\delta \rho\right) \cdot \cdot a^{i j}\left(\rho_{j} \otimes n\right) v_{, i}\right) d s-\int_{s} N\left(\delta r_{s}-\delta \rho\right) \cdot h^{i j}\left(\rho_{i} \otimes \rho_{j}\right)\left(v_{s}-v\right) d s
\end{gathered}
$$




\subsection{Weak Formulation for the Curve-To-Curve Contact Pair}

Since in this type of contact master and slave parts are equivalent, the virtual work can be equivalently written as an integral either along the first curve, or along the second curve and, therefore, for symmetry reasons the following expression is taken:

$$
\begin{gathered}
\delta W=\operatorname{Sym}\left\{R_{1} \cdot\left(\delta \rho_{1}-\delta \rho_{2}\right)\right\}=-\operatorname{Sym}\left\{T_{1} \sqrt{\left(1-r k_{1} \cos \varphi_{1}\right)^{2}+\left(r \varkappa_{1}\right)^{2}} \delta s_{1}+N \delta r+M_{1} \delta \varphi_{1}\right. \\
\left.+T_{1} \frac{\varkappa_{1} r^{2}}{\sqrt{\left(1-r k_{1} \cos \varphi_{1}\right)^{2}+\left(r \varkappa_{1}\right)^{2}}} \delta \varphi_{1}+M_{1} \varkappa_{1} \delta s_{1}\right\},
\end{gathered}
$$

with corresponding normal $N$, tangential $T_{i}$ components and circumferential moments. Details of linearization are given in [8].

\subsection{Weak Formulation for the Curve-To-Surface Contact Pair}

\subsubsection{A Special Combination of Curve-To-Curve and Segment-To-Analytical-Surface Algorithm}

Considering the developed kinematics for the Curve-To-Surface interaction, we are formulating the weak form in the curve Serret-Frenet coordinate system as an integral along the curve $l$. It is formulated via parameters expressed either in the curve Serret-Frenet coordinate system; i.e. or via parameters defined in the Gaussian surface coordinate system. Starting with the weak form expressed at the curve, we have:

$$
\begin{gathered}
\delta W=\int_{l} R_{s} \cdot\left(\delta r_{s}-\delta \rho\right) d l= \\
-\int_{l}\left(\delta u_{e} N+\delta u_{\tau} T+\delta u_{g} G\right)=\int_{l}\left(T\left(\delta r_{s}-\delta \rho\right) \cdot \tau+N\left(\delta r_{s}-\delta \rho\right) \cdot e+G\left(\delta r_{s}-\delta \rho\right) \cdot g\right) d l \quad \text { (via curve parameters) } \\
\int_{l}\left(T\left(\delta r_{s}-\delta \rho\right) \cdot \tau+N\left(\delta r_{s}-\delta \rho\right) \cdot e+G\left(\delta r_{s}-\delta \rho\right) \cdot g\right) d l
\end{gathered}
$$

(or via surface parameters)

$$
\begin{gathered}
=\int_{l}\left[N\left(\delta r_{s}-\delta \rho\right) \cdot e+T\left(\rho_{i} \cdot \tau\right) \delta \xi^{i}+G\left(\rho_{i} \cdot \mathrm{g}\right) \delta \xi^{i}\right] d l= \\
=\int_{l}\left[N\left(\delta r_{s}-\delta \rho\right) \cdot e-\left(T^{j} \rho^{i} \cdot\left(\delta r_{s}-\delta \rho\right)\right) \rho_{i} \cdot(\tau \otimes \tau+g \otimes g) \rho_{j}\right] d l .
\end{gathered}
$$

The linearization results will combine the linearization for both Surface-To-Surface and Curve-To-Curve contact pairs, discussed in detail in.

\subsubsection{A Special Consideration in the Darboux Basis}

In this special case the Curve-To-Surface contact pair can be regarded as the equilibrium of ropes on orthotropic rough. The weak form is written as, see derivation details in [11]

$$
\delta W_{c}=\int_{0}^{l}\left(T \delta \varepsilon+\left(\tilde{T}+q_{\tau}\right) \delta u_{\tau}+\left(k_{g} T+G+q_{g}\right) \delta u_{g}+\left(N+k_{n} T+q_{n}\right) \delta u_{n}\right) d s
$$

Computationally, values of pulling $\tilde{T}$ and dragging $G$ forces can be evaluated employing a return-mapping algorithm based on the dependence on the constitutive equation for the frictional force:

$$
\Phi:=\sqrt{\frac{\tilde{T}^{2}}{\mu_{\tau}^{2}}+\frac{G^{2}}{\mu_{g}^{2}}}<|N|
$$

The advantages of the description in the Darboux basis are:

- contact kinematics is precisely described;

- an anisotropic friction law for the rope-surface interaction can be easily incorporated;

- new analytical solutions such as a generalized Euler-Eytelwein formula can be obtained.

For the last statement, we represent here result of the theorem for the equilibrium of a rope, see details in [11], which is amazingly a generalization of the famous Euler-Eytelwein formula, or belt friction formula for the limit load known as $T=T_{0} e^{\mu \varphi}$ (here $\varphi$ is the angular coordinate of a rope on $2 \mathrm{D}$ circular cylinder) published by Euler in [13] in the year 1769. 
Theorem. If a rope loaded by tangential forces is laying in equilibrium on a rough orthotropic surface then three following conditions (all of them) are satisfied:

1) No separation—normal reaction $\$ N \$$ is positive for all points of the curve:

$$
N=-k_{n} T>0
$$

2) Dragging coefficient of friction $\mu_{g}$ and angle $\alpha$ are satisfying the following criteria for all points of the curve

$$
-\mu_{g}<\tan \alpha<+\mu_{g}
$$

3) Limit values of the tangential forces:

The forces at both ends $T$ and $T_{0}$ are satisfying the following inequality

$$
\begin{gathered}
T_{0} e^{-\int_{s} \omega d s}<T<T_{0} e^{\int_{s} \omega d s} \\
\text { with } \omega=\mu_{\tau} \sqrt{k_{n}^{2}-\frac{k_{g}^{2}}{\mu_{g}^{2}}}=\mu_{\tau} k \sqrt{\cos ^{2} \alpha-\frac{\sin ^{2} \alpha}{\mu_{g}^{2}}}
\end{gathered}
$$

\section{Application of the Geometrically Exact Contact for Finite Cell Method (FCM)}

The Finite Cell Method (FCM) provides a method for the computation of structures which can be described as a mixture of high-order FEM and a special integration technique, see [14]. The method is one of the novel computational methods and is highly developed within the last decade. One of the major problems of FCM is the description of boundary conditions inside cells as well as in sub-cells. And a completely open problem is the description of contact. Therefore, the motivation of the current work is to develop a set of computational contact mechanics approaches which will be effective for the Finite Cell Method.

Alternative contact approaches for FCM based on the application of the geometrically exact contact theory.

The following alternative contact algorithms are developed and tested:

- CSTAS contact element (Cell-Surface-To-Analytical-Surface) for contact with rigid bodies;

- DCTC contact element (Discrete-Cell-To-Cell)-based on the representation of the integration point as a discrete finite element for both deformable bodies.

\subsection{Cell-Surface-To-Analytical-Surface (CSTAS) Contact Approach}

In this approach a special contact element cell-wisely, namely, the contact element is developed based on the exact boundary inside the sub-domain/sub-cell, see more detail in [15]. This requires transformations inside each sub-domain/sub-cell, which will be illustrated using the Hertz problem. The main idea of the CSTAS contact approach is the building of a linear contact element exactly on the contact boundary inside each sub-domain/ sub-cell following Surface-To-Surface contact pair, see Figure 6. This contact element has its own parameterization, though it is defined in the coordinate system of the finite element cell-wisely.
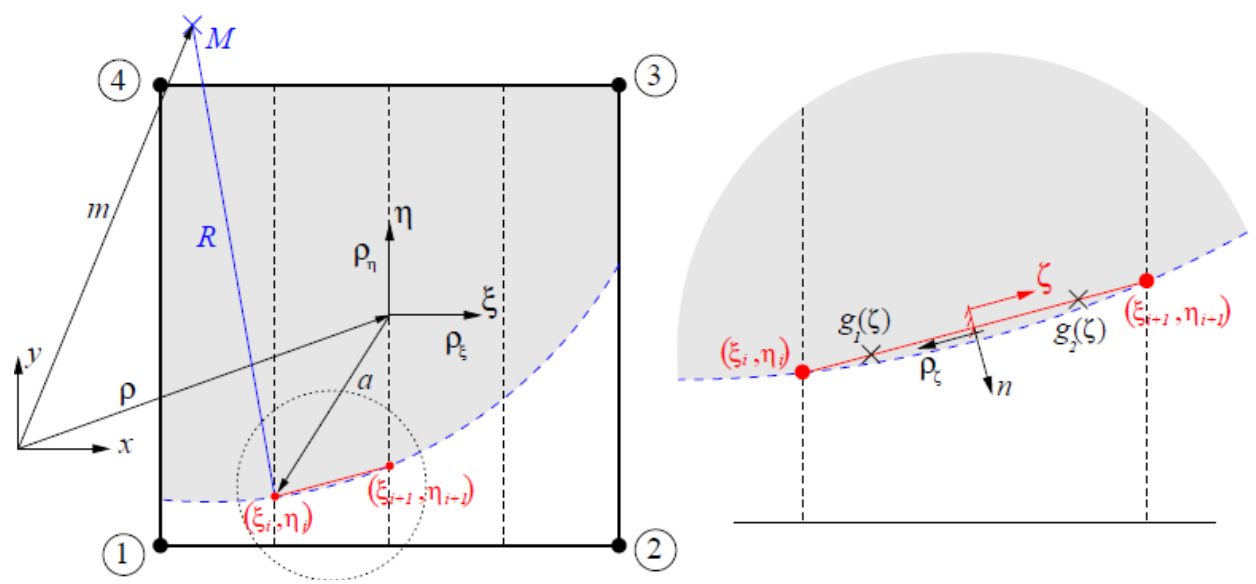

Figure 6. Cell-wise structure of the Cell-To-Analytical Surface contact element. 
In order to increase the performance of the cell-wise application the adaptive cell refinement with CSTAS method is applied. Fairly high dense mesh with low order of approximation: $20 \times 20$ finite elements of 2th order with additional $10 \times 10$ cell subdivision of the bounding area (more dense area in the Figure 7 with a corresponding CSTAS contact element, see the representation of the mesh by integration points in Figure 7 on the left side and medium dense mesh with higher order of approximation: $8 \times 8$ finite cell elements of 5 th order (integrated Legendre polynomials) with additional $10 \times 10$ cell subdivision of the bounding area (more dense area in the Figure) with a corresponding CSTAS contact element, see the representation of the mesh by integration points in Figure 7 on right side are compared with the analytical Hertz solution.

\subsection{Discrete Cell to Cell Contact (DCTC) as the Simplest Contact Algorithm}

The simplest implementation for contact between deformable bodies can be constructed based on the nature of FCM - a set of Gauss points is represented as discrete elements and then a contact algorithm used for the Finite Discrete Method (FDM) is employed. The idea of the DCTC method is presented in Figure 8 based on the assumption that a rather dense cell subdivision is employed. In this case, each cell is embedded in a circle with the radius $\chi$, allowing a ball-to-ball discrete contact, or (a Discrete-Cell-To-Cell) method. The contact radius $\chi$

should be adjusted with regards to the distance between the neighboring integration points. By the selection of the value for the radius $\chi$ (here the size of cells and corresponding radius for contacting bodies are taken into account) the following facts should be taken into account:

- Convergence of the solution is better if the radius is larger;

- The radius $\chi$ should not be too large in order to avoid overlapping of the nearest integration points;

- The radius $\chi$ should not be too small in order to avoid loss of contact, if one circle (ball) would slide between other two without contact.

The DCTC contact approach allows to describe efficiently the self-contact in a pore, see in Figure 9.
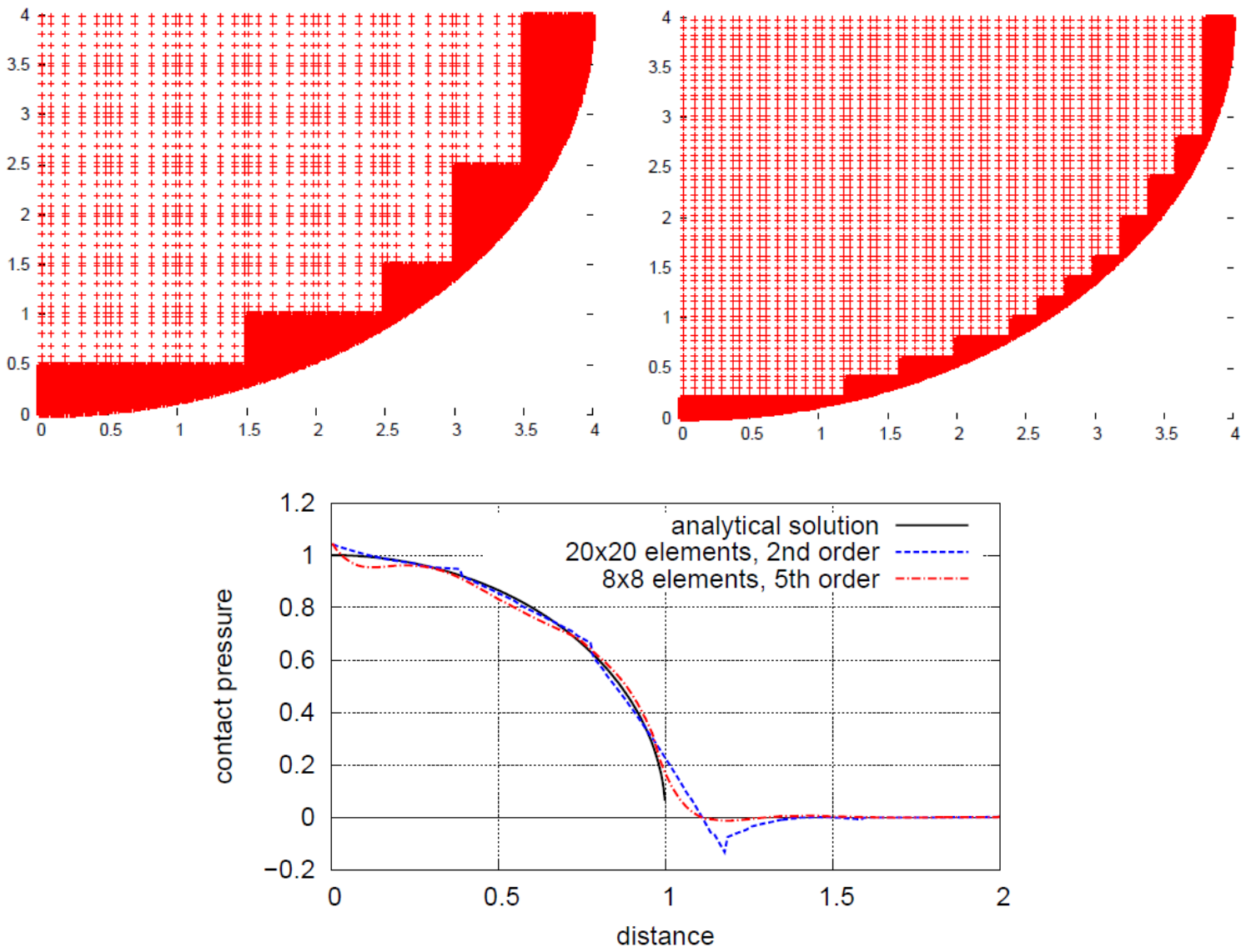

Figure 7. Cell refinement for the CTAS contact approach for the Hertz problem. 


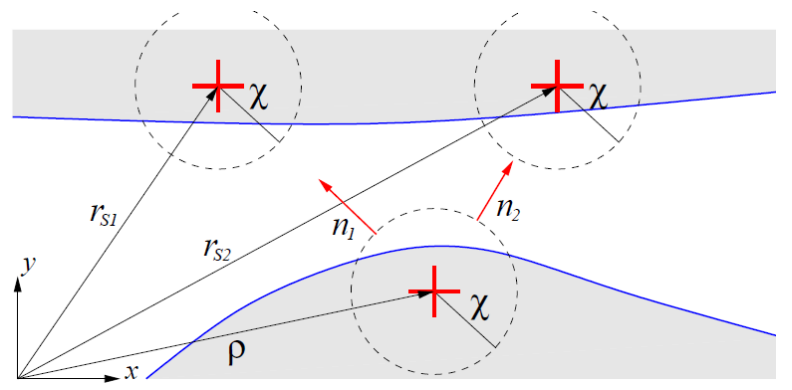

Figure 8. Representation of the discrete cell to cell contact approach.
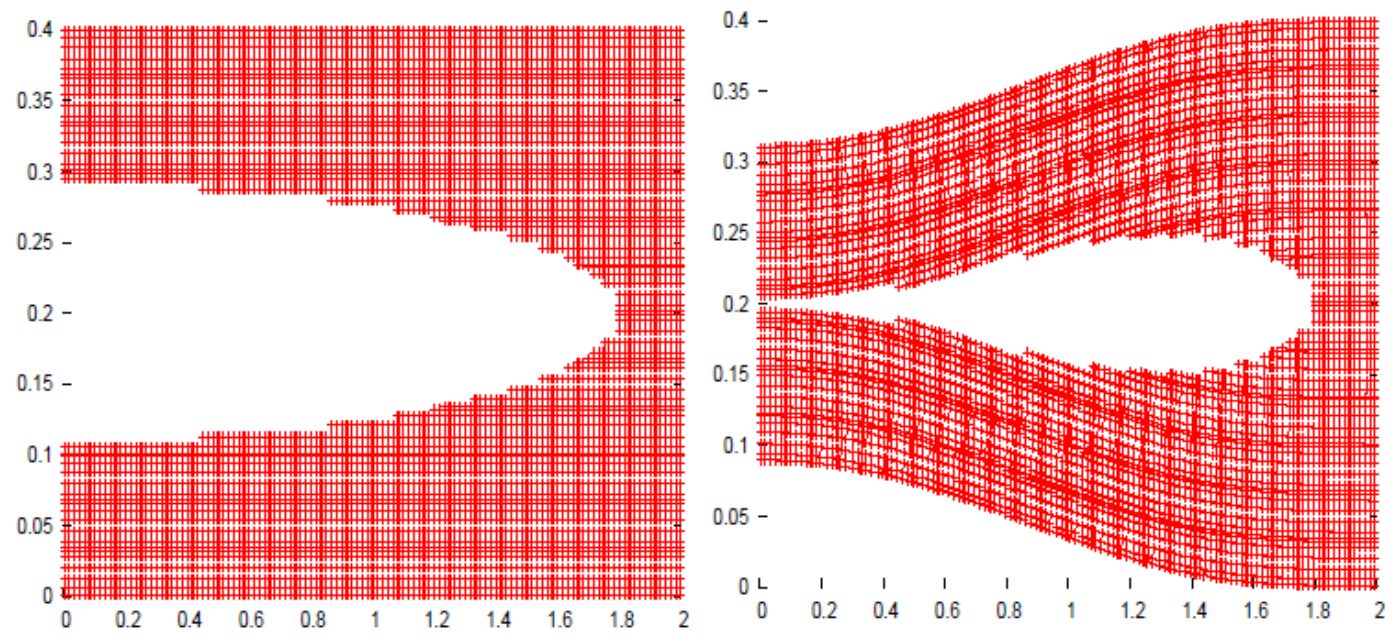

Figure 9. Selfcontact in a pore, Discrete Cell-To-Cell contact approach.

\section{Conclusion}

The geometrically exact theory for contact interaction allows to describe and to construct computational algorithm for all available geometrical contact pairs Surface-To-Surface, Curve-To-Curve, Curve-To-Surface etc. as well as allows to construct computational contact algorithms not only for the finite element method, but also for other methods e.g. Finite Cell Method.

\section{References}

[1] Laursen, T.A. (2002) Computational Contact and Impact Mechanics. Fundamentals of Modeling Interfacial Phenomena in Nonlinear Finite Element Analysis. Springer, Berlin, 454.

[2] Wriggers, P. (2006) Computational Contact Mechanics. 2nd Edition, Springer, Berlin, 520.

http://dx.doi.org/10.1007/978-3-540-32609-0

[3] Litewka, P. (2010) Finite Element Analysis of Beam-to-Beam Contact. Springer, Berlin, 160. http://dx.doi.org/10.1007/978-3-642-12940-7

[4] Konyukhov, A. and Schweizerhof, K. (2013) Computational Contact Mechanics-Geometrically Exact Theory for Arbitrary Shaped Bodies. Springer, Heidelberg, 444.

[5] Konyukhov, A. and Izi, R. (2015) Introduction into Computational Contact Mechanics: A Geometricall Approach. Wiley, 304.

[6] De Lorenzis, L., Wriggers, P. and Hughes, T.J.R. (2014) Isogeometric Contact a Review. GAMM Mitteilungen, 37, 85-123. http://dx.doi.org/10.1002/gamm.201410005

[7] Konyukhov, A. and Schweizerhof, K. (2008) On the Solvability of Closest Point Projection Procedures in Contact Analysis: Analysis and Solution Strategy for Surfaces of Arbitrary Geometry. Comput. Method Appl. M., 197, 30453056. http://dx.doi.org/10.1016/j.cma.2008.02.009

[8] Konyukhov, A. and Schweizerhof, K. (2010) Geometrically Exact Covariant Approach for Contact between Curves. 
Comput. Method Appl. M., 199, 2510-2531. http://dx.doi.org/10.1016/j.cma.2010.04.012

[9] Konyukhov, A. and Schweizerhof, K. (2012) Geometrically Exact Theory for Contact Interactions of 1D Manifolds. Algorithmic Implementation with Various Finite Element Models. Comput. Methods in Appl. M., 205-208, 130-138.

[10] Konyukhov, A. and Schweizerhof, K. (2015) On Some Aspects for Contact with Rigid Surfaces: Surface-to-Rigid Surface and Curves-to-Rigid Surface Algorithms. Comput. Methods in Appl. M., 283, 74-105.

[11] Konyukhov, A. (2013) Contact of Ropes and Orthotropic Rough Surfaces. ZAMM, Z. Angew. Math. Mech., 1-18.

[12] Konyukhov, A. and Schweizerhof, K. (2005) Covariant Description for Frictional Contact Problems. Comput. Mech., 35, 190-213. http://dx.doi.org/10.1007/s00466-004-0616-7

[13] Euler, L. (1769) Remarques sur l'effet du frottement dans l'equilibre. Memoires de l'Academie des Sciences de Berlin, 18, 265-278.

[14] Düster, A., Parzivian, J., Yang, Z. and Rank, E. (2008) The Finite Cell Method for Three-Dimensional Problems of Solid Mechanics. Comput. Methods in Appl. M., 197, 3768-3782.

[15] Konyukhov, A., Lorenz, Ch. and Schweizerhof, K. (2015) Various Contact Approaches for the Finite Cell Method, Computational Mechanics. http://dx.doi.org/10.1007/s00466-015-1174-x 